\title{
Identification of tomato plant as a novel host model for Burkholderia pseudomallei
}

\author{
Yian Hoon Lee ${ }^{1}$, Yahua Chen ${ }^{1}$, Xuezhi Ouyang ${ }^{3}$, Yunn-Hwen Gan ${ }^{1,2^{*}}$
}

\begin{abstract}
Background: Burkholderia pseudomallei is the causative agent for melioidosis, a disease with significant mortality and morbidity in endemic regions. Its versatility as a pathogen is reflected in its relatively huge $7.24 \mathrm{Mb}$ genome and the presence of many virulence factors including three Type Three Secretion Systems known as T3SS1, T3SS2 and T3SS3. Besides being a human pathogen, it is able to infect and cause disease in many different animals and alternative hosts such as C. elegans.

Results: Its host range is further extended to include plants as we demonstrated the ability of B. pseudomallei and the closely related species $B$. thailandensis to infect susceptible tomato but not rice plants. Bacteria were found to multiply intercellularly and were found in the xylem vessels of the vascular bundle. Disease is substantially attenuated upon infection with bacterial mutants deficient in T3SS1 or T3SS2 and slightly attenuated upon infection with the T3SS3 mutant. This shows the importance of both T3SS1 and T3SS2 in bacterial pathogenesis in susceptible plants.
\end{abstract}

Conclusions: The potential of B. pseudomallei as a plant pathogen raises new possibilities of exploiting plant as an alternative host for novel anti-infectives or virulence factor discovery. It also raises issues of biosecurity due to its classification as a potential bioterrorism agent.

\section{Background}

Burkholderia pseudomallei is a Gram-negative bacterium that is the causative agent for melioidosis, a disease endemic in Southeast Asia and Northern Australia with significant morbidity and mortality [1,2]. The bacterium exhibits broad host range and has been shown to cause disease in cattle, pigs, goats, horses, dolphins, koalas, kangaroos, deers, cats, dogs and gorillas [3]. Acquisition of the bacterium could be through inhalation of aerosol, ingestion of contaminated water and inoculation through open skin [4]. In humans, the disease could present with varied manifestations ranging from asymptomatic infection, localized disease such as pneumonia or organ abscesses to systemic disease with septicemia [5]. The disease could be acute or chronic, and relapse from latency is possible [6].

The versatility of $B$. pseudomallei as a pathogen is reflected in its huge $7.24 \mathrm{Mb}$ genome organized into two chromosomes [7]. One of the most important

\footnotetext{
* Correspondence: bchganyh@nus.edu.sg

'Department of Biochemistry, Yong Loo Lin School of Medicine, National University of Singapore, 8 Medical Drive, 117597, Singapore
}

virulence factors that has been partially characterized in B. pseudomallei is its Type Three Secretion Systems (T3SS), of which it has three $[8,9]$. Each T3SS typically consists of a cluster of about 20 genes encoding structural components, chaperones and effectors which assemble into an apparatus resembling a molecular syringe that is inserted into host cell membrane for the delivery of bacterial effectors into host cell cytosol. One of the B. pseudomallei T3SS known as Bsa or T3SS3 resembles the inv/mxi/spa T3SS of Salmonella and Shigella, and has been shown to be important for disease in animal models [10]. The other two T3SS (T3SS1 and 2) resemble the T3SS of plant pathogen Ralstonia solanacearum [11] and do not contribute to virulence in mammalian models of infection [12]. Being a soil saprophyte and having the plant pathogen-like T3SS raise the possibility that $B$. pseudomallei could also be a plant pathogen. As $B$. pseudomallei is a risk group 3 agent with specific requirements for containment, we first test this hypothesis using the closely related species $B$. thailandensis as a surrogate model especially in experiments where risk of aerosolization is high, before we verify key 
experiments with $B$. pseudomallei. B. thailandensis is considered largely avirulent in mammalian hosts unless given in very high doses $[13,14]$. We infected both tomato as well as rice plants with $B$. pseudomallei to determine their susceptibility to disease. Furthermore, the role of the three $B$. pseudomallei T3SS in causing plant disease is evaluated and the implication of the ability of B. pseudomallei to infect plants is discussed.

\section{Methods}

\section{Bacterial strains, plasmids and growth conditions}

All bacterial strains, plasmids used and constructed are listed in Table 1 . All strains of $B$. thailandensis and $B$. pseudomallei were cultured at $37^{\circ} \mathrm{C}$ in Luria-Bertani (LB) medium or on Tryptone Soy Agar (TSA) plates. To obtain log-phase culture, $250 \mu \mathrm{L}$ of overnight culture was inoculated into $5 \mathrm{~mL} \mathrm{LB}$ medium and cultured for 2.5 hours with constant shaking at $100 \mathrm{rpm}$. Escherichia coli strains were cultivated at $37^{\circ} \mathrm{C}$ in LB medium. Antibiotics were added to the media at the following final concentrations of $100 \mu \mathrm{g} / \mathrm{mL}$ (ampillicin); $25 \mu \mathrm{g} / \mathrm{mL}$ (kanamycin); $10 \mu \mathrm{g} / \mathrm{mL}$ (tetracycline); and $25 \mu \mathrm{g} / \mathrm{mL}$ (zeocin) for E. coli, $250 \mu \mathrm{g} / \mathrm{mL}$ (kanamycin); $40 \mu \mathrm{g} / \mathrm{mL}$ (tetracycline); $25 \mu \mathrm{g} / \mathrm{mL}$ (gentamicin) and $1000 \mu \mathrm{g} / \mathrm{mL}$ (zeocin) for B. pseudomallei. All antibiotics were purchased from Sigma (St Louis, MO, USA).

\section{Plant material}

Tomato seeds of the Solanum lycopersicum variety Season Red F1 Hybrid (Known-You Seeds Distribution (S.E. A) Pte Ltd) and Arabidopsis thaliana (Loh Chiang Shiong, NUS) were surface sterilized with $15 \%$ bleach solution for 15 minutes with vigorous shaking. The seeds were rinsed in sterile distilled water and germinated in MS agar medium. The seedlings were cultivated with a photoperiod of 16 hour daylight and 8 hour darkness. One month old plantlets were used for infection. Tomato plantlets were transferred into $50 \mathrm{~mL}$ Falcon tubes with $5 \mathrm{~mL}$ of liquid MS medium for infection while $1 \mathrm{~mL}$ of MS medium was used for Arabidopsis. Rice seeds (Japonica nipponbare) were obtained from Dr Yin Zhong Zhao (Temasek Life Sciences Laboratories, Singapore). Seeds were surface sterilized as described above. The seeds were rinsed in sterile distilled water and germinated in N6 agar medium. The germinated seedlings were placed on N6 agar supplemented with $2 \mathrm{mg} / \mathrm{mL}$ of 2, 4-dichlorophenyoxyacetic acid $(2,4-D)$ in the dark to induce callus production. The callus were regenerated on N6 medium

Table 1 All bacterial strains, plasmids used and constructed.

\begin{tabular}{|c|c|c|}
\hline Name & Description & Source or Reference \\
\hline pK18mobsacB & oriT; $\mathrm{Km}^{\mathrm{R}}$; sacB gene & [32] \\
\hline pGEM-tet & pGEM containing a tetracycline resistance cassette, Tet $^{R}, \mathrm{Amp}^{\mathrm{R}}$ & Y. Chen, unpublished \\
\hline pCLOXZ1 & pGEM containing a zeocin resistance cassette, Zeo ${ }^{R}, A_{m p}^{R}$ & Y. Chen, unpublished \\
\hline $\begin{array}{l}\text { pT3SS1/upstream/downstream/ } \\
\text { tet }\end{array}$ & $\begin{array}{l}\text { pK18mobsacB containing upstream and downstream of TTSS1 flanking a tet cassette, } \\
\mathrm{Km}^{R}, \text { Tet }^{R}\end{array}$ & This study \\
\hline $\begin{array}{l}\text { pT3SS2/upstream/downstream/ } \\
\text { tet }\end{array}$ & $\begin{array}{l}\text { pK18mobsacB containing upstream and downstream of TTSS2 flanking a tet cassette, } \\
\mathrm{Km}^{R}, \text { Tet }^{R}\end{array}$ & This study \\
\hline $\begin{array}{l}\text { pT3SS3/upstream/downstream/ } \\
\text { zeo }\end{array}$ & $\begin{array}{l}\text { pK18mobsacB containing upstream and downstream of TTSS3 flanking a zeo cassette, } \\
\mathrm{Km}^{R}, \mathrm{ZeO}^{\mathrm{R}}\end{array}$ & This study \\
\hline \multicolumn{3}{|l|}{ E. coli } \\
\hline $\mathrm{DH} 5 \alpha$ & Infection strain & Lab stock \\
\hline TG1 & Cloning host & Zymo Research \\
\hline SM10גpir & Conjugation strain & {$[33]$} \\
\hline \multicolumn{3}{|l|}{ B. thailandensis } \\
\hline ATCC700388 & & ATCC \\
\hline \multicolumn{3}{|l|}{ B. pseudomallei } \\
\hline K96243 & Clinical isolate & Thailand \\
\hline 561 & Kangaroo isolate & $\begin{array}{l}\text { Eu Hian Yap, } \\
\text { unpublished }\end{array}$ \\
\hline 612,490 & Avian isolates & $\begin{array}{l}\text { Eu Hian Yap, } \\
\text { unpublished }\end{array}$ \\
\hline 77/96, 109/96 & Soil isolates & $\begin{array}{l}\text { Eu Hian Yap, } \\
\text { unpublished }\end{array}$ \\
\hline KHW & Wild-type parental strain, clinical isolate, $\mathrm{Km}^{\mathrm{S}}$ & {$[20]$} \\
\hline KHWDT3SS1 & BPSS1386-1411 region was replaced with tet cassette, $\mathrm{Tet}^{\mathrm{R}}, \mathrm{Km}^{\mathrm{S}}$ & This study \\
\hline KHW $\triangle \mathrm{T} 3 \mathrm{SS} 2$ & BPSS1592-1629 region was replaced with tet cassette, Tet $^{R}, \mathrm{Km}^{\mathrm{S}}$ & This study \\
\hline KHW $\triangle \mathrm{T} 3 \mathrm{SS} 3$ & BPSS1520-1552 region was replaced with zeo cassette, Zeo ${ }^{R}, \mathrm{Km}^{S}$ & This study \\
\hline
\end{tabular}


supplemented with $2 \mathrm{mg} / \mathrm{mL}$ Benzylaminopurine (BA), 1 $\mathrm{mg} / \mathrm{mL}$ Naphthylacetic Acid (NAA), $1 \mathrm{mg} / \mathrm{mL}$ Indole-3acetic acid (IAA) and $1 \mathrm{mg} / \mathrm{mL}$ Kinetin under 16 hour daylight and 8 hour dark photoperiod. Rice plantlets were transferred and maintained in MS agar medium. The plantlets were transferred into $50 \mathrm{~mL}$ Falcon tubes with $5 \mathrm{~mL}$ of liquid MS medium for infection. Some plantlets were also wounded by cutting off the roots before being transferred.

\section{Plant infection}

Tomato, rice and Arabidopsis plantlets were infected with $\log$ phase cultures at the concentration of $1 \times 10^{7}$ colony forming units (cfu)/5 mL medium by immersing only the roots of the plantlets in the inoculum in a $50 \mathrm{~mL}$ tube. The plantlets were maintained at $24-25^{\circ}$ $\mathrm{C}$, shaking at $100 \mathrm{rpm}$. The plantlets were observed for symptoms such as yellowing of leaves, blackening of the leaf veins, wilting and necrosis daily over 7 days. Each plantlet was scored daily on a disease index score of 1 to 5 based on how extensive the symptoms were as calculated by the percentage of the plant with symptoms (1: no symptoms; $2: 1$ to $25 \%$ of the plant showed symptoms; 3: 26 to $50 \%$ of the plant showed symptoms; $4: 51$ to $75 \%$ of the plant showed symptoms; 5 : 76 to $100 \%$ of the plant showed symptoms or the plant was dead) [15]. Each experiment included at least 12 to 20 plantlets infected with bacteria except for experiments with rice and Arabidopsis plantlets where 6 plantlets were used. All experiments were repeated at least twice.

Multiplication of $B$. thailandensis in tomato plantlets and leaves

Tomato plantlets were infected with bacteria through unwounded roots and three leaves from each plantlet were excised at day $1,3,5$ and 7 after infection. The leaves were macerated in $1 \mathrm{~mL}$ PBS with a micro-pestle, serially diluted and plated on TSA plates in duplicates. Tomato leaves were infected by cutting with a pair of scissors dipped in $1 \times 10^{9} \mathrm{cfu} / \mathrm{mL}$ of $B$. thailandensis. Five plantlets were used in each experiment. At days 1 and 3 after infection, one infected leaf from each plantlet was excised, washed with $10 \%$ bleach solution for $1 \mathrm{~min}$ and rinsed with sterile water. The leaf was blotted dry on sterile filter paper and imprinted on TSA agar plates to determine if there were any bacteria on the surface of the leaves. The imprinted plates were incubated at $37^{\circ} \mathrm{C}$ for 24 hours before checking for any bacteria growth. The leaves were then weighed and macerated in $1 \mathrm{~mL}$ PBS with a micro-pestle, serially diluted and plated on TSA plates in duplicates. Only leaf samples which did not show any bacteria growth on the imprinted plates will be counted to avoid counting contaminating bacteria from leaf surfaces.

\section{Transmission Electron Microscope (TEM)}

Tomato leaf and rice blade were infected by cutting with a pair of scissors dipped in $1 \times 10^{9} \mathrm{cfu} / \mathrm{mL}$ of $B$. pseudomallei strain $\mathrm{KHW}$ or $B$. thailandensis. One day after infection, the infected tomato leaf and rice blade were excised for TEM. One millimeter from the infected leaf/ blade edge were cut and discarded to avoid contamination from extracellular bacteria at the infection site. A further two millimeter from the infected leaf/blade edge were then cut and sliced into smaller sections and fixed with $4 \%$ glutaraldehyde in $0.1 \mathrm{M}$ phosphate buffer under vacuum for 4 hours. It was post-fixed with $1 \%$ osmium tetroxide in $0.1 \mathrm{M}$ phosphate buffer for 1 hour at $4^{\circ} \mathrm{C}$. Samples were dehydrated sequentially through $30 \%$, $50 \%, 70 \%, 90 \%, 100 \%$ ethanol, and finally in propylene oxide prior to infiltration with Spurr resin [16]. Samples were embedded in $100 \%$ spur resin and polymerized at $70^{\circ} \mathrm{C}$ overnight. Ultra-thin sections were cut on a Leica Ultracut UCT ultra-microtome and examined with a transmission electron microscope (JEM1230, JEOL, Japan) at $120 \mathrm{kV}$.

\section{Growth of bacteria in different media}

Overnight cultures were used to inoculate $5 \mathrm{~mL}$ of $\mathrm{LB}$ and Murashige and Skoog (MS) [17] medium to a starting optical density at $600 \mathrm{~nm}$ of 0.1 . The cultures were incubated at $37^{\circ} \mathrm{C}$ for $\mathrm{LB}$ medium and $25^{\circ} \mathrm{C}$ for MS medium. Optical density at $600 \mathrm{~nm}$ for all cultures was measured at 0, 2.5, 6 and 24 hours. All experiments were repeated twice with duplicates.

\section{Generation of B. pseudomallei T3SS1, T3SS2 and T3SS3} mutants

Approximate one $\mathrm{kb}$ fragments upstream and downstream of the T3SS1, T3SS2 or T3SS3 locus were amplified from $B$. pseudomallei KHW genomic DNA and subsequently cloned into pK18mobsacB. The tet cassette from pGEM-tet or zeo cassette (kindly provided by Dr Herbert Schweizer, Colorado State University, USA) from pCLOXZ1 was inserted between the upstream and downstream fragments resulting in pT3SS1/upstream/ downstream/tet, pT3SS2/upstream/downstream/tet, and pT3SS3/upstream/downstream/zeo. The plasmids were electroporated into SM10 conjugation host and conjugated into B. pseudomallei strain KHW. Homologous recombination was selected for retention of antibiotic marker (Tet or Zeo) linked to the mutation and loss of the plasmid marker $(\mathrm{Km})$ to generate KHW $\mathrm{T} 3 \mathrm{SS} 1$, KHW $\Delta$ T3SS2 and KHW $\Delta$ T3SS3. Each mutant was confirmed by PCR for the loss of a few representative T3SS genes in the locus.

\section{Cytotoxicity assay on THP-1 cells}

Human monocytic cell line THP-1 were maintained in RPMI 1640 (Sigma), supplemented with 10\% Fetal Calf Serum (FCS, Hyclone Laboratories, Logan, UT), 200 $\mathrm{mM}$ L-glutamine, $100 \mathrm{Unit} / \mathrm{mL}$ penicillin and $100 \mu \mathrm{g} /$ 
$\mathrm{mL}$ streptomycin. THP-1 cells were seeded at a concentration of $1 \times 10^{6}$ cells per $100 \mu \mathrm{L}$ in 96 -well plate in medium without FCS and antibiotics. Log phase bacteria were used for infection at multiplicity of infection (MOI) of 100:1. Kanamycin $(250 \mu \mathrm{g} / \mathrm{mL})$ was added one hour after infection to suppress the growth of extracellular bacteria. Supernatant was collected 6 hours after infection. Lactate dehydrogenase (LDH) activity in the supernatant was measured with the Cytotoxicity Detection Kit (Roche) according to manufacturer's instruction. Percentage cytotoxicity was calculated by the formula:

$$
\% \text { cytotoxicity }=\frac{\text { Sample } \mathrm{LDH} \text { release }- \text { spontaneous release }}{\text { Maximum release }- \text { spontaneous release }} \times 100
$$

\section{Statistical analysis}

Average disease scores with standard deviation were calculated based on at least 100 tomato plantlets infected with each strain of bacteria or mutant. Data were analyzed using repeated measure analysis of variance [18]. All statistical analyses were performed using SPSS version 17 software (SPSS Inc). A $p$ value of less than 0.001 is considered significant.

\section{Results \\ Using B. thailandensis infection of tomato plantlets as a model}

To mimic infection via a possible natural route, the unwounded roots of tomato plantlets were immersed in media inoculated with $1 \times 10^{7} \mathrm{cfu}$ of bacteria. Only the roots were in contact with the inoculum. Tomato plantlets infected via the roots by $B$. thailandensis showed progressive symptoms such as yellowing of leaves, blackening of the leaf veins, wilting and necrosis whereas uninfected plantlets remained healthy and did not show any disease symptoms throughout the period (Fig 1A-B). Most infected plantlets were dead on day 7. All plantlets were monitored over a period of seven days. Disease was scored daily for every plantlet on an index from 1-5 based on the extent of symptoms presented as described in Methods. The average disease score for a particular day represent the mean disease scores for all the plantlets with the same treatment on that day. As infection progressed over time, the average disease score for $B$. thailandensis-infected plants increased progressively, reaching a maximum disease score of 5 on day 7 (Fig $1 C)$. In contrast, plantlets infected with $E$. coli in the same manner via the roots showed a slight progression of average disease scores over time and reached a maximum disease score of 2 on day 7 (Fig 1C), demonstrating that the extensive disease and death seen was specific to $B$. thailandensis infection and not due to non-specific stress induced by the experimental manipulations.
For a phytopathogen to successfully colonize the plant, it must be able to replicate intercellularly [19]. To determine whether bacteria are able to replicate intercellularly, we sampled leaves from two representative plantlets which had been inoculated with bacteria via unwounded roots at 1, 3, 5 and 7 days post-inoculation. Three leaves were sampled at each time-point per plantlet. Both plantlets showed a progressive increase in bacterial load in their leaves over time (Fig 1D).

\section{Susceptibility of tomato plantlets to $B$. pseudomallei infection}

Having established that $B$. thailandensis can infect tomato plantlets and cause disease, we determine whether B. pseudomallei would similarly infect tomato plantlets. We included strains isolated from humans, animals or the environment such as two clinical isolates (K96243 and KHW), a kangaroo isolate 561, two bird isolates (612 and 490) and two soil isolates (77/96 and 109/96) on their ability to infect tomato plants. B. pseudomallei is able to infect tomato plantlets to a similar degree as $B$. thailandensis with almost identical disease symptoms. All isolates were able to infect and cause disease to a similar extent (Fig 2), showing that the ability to infect susceptible plants is unlikely to be strainspecific.

\section{Localization of bacteria at site of infection}

Having established the ability of both B. thailandensis and $B$. pseudomallei to be phytopathogens capable of infecting tomato plants, we next examined the localization of the bacteria upon inoculation into the leaf via TEM. We first examined whether bacteria inoculated into the leaves were able to survive and replicate. To ensure that there were no bacteria on the leaf surfaces, the leaves were surface sterilized with bleach and washed in sterile water before weighing and maceration. $B$. thailandensis was able to replicate in the leaves after inoculation (Fig 3A). The number of bacteria increased by about 10 fold three days after infection although the numbers did not reach statistical significance by the student $t$ test ( $\mathrm{p}>0.05)$. When examined under TEM, $B$. pseudomallei and $B$. thailandensis could be found in the xylem of the vascular bundle of the inoculated leaf (Fig $3 \mathrm{~B}-\mathrm{C})$. The rest of the surrounding cells were not colonized, suggesting that the bacteria spread to the rest of plant through the xylem vessels.

\section{The role of T3SS in plant infection}

To determine the role of T3SS in plant infection, we created $B$. pseudomallei deletion mutants lacking the entire region of T3SS1, T3SS2 or T3SS3 in strain KHW (Table 1). We first examined these mutants in the established macrophage cytotoxicity model and confirmed the necessity of T3SS3 in mediating cytotoxicity [20] whereas mutants losing T3SS1 and T3SS2 were as cytotoxic as wildtype bacteria to THP-1 cells (Fig 4A). This 
A

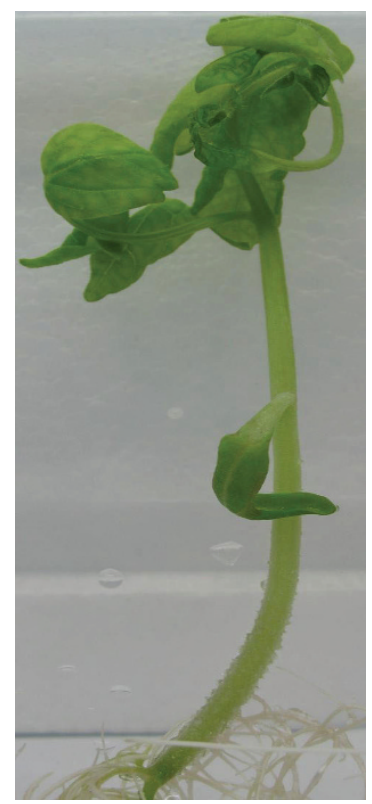

B

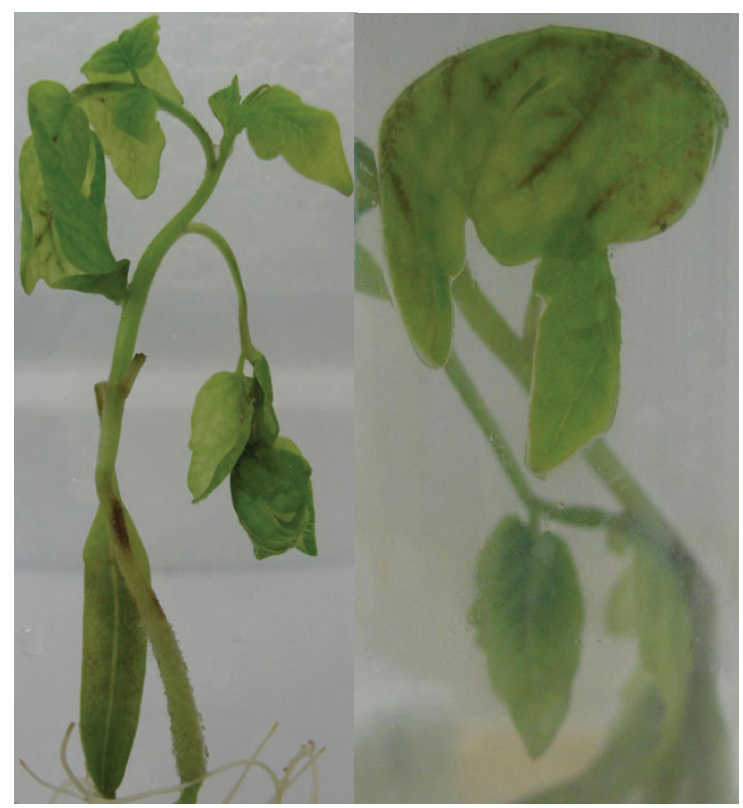

C

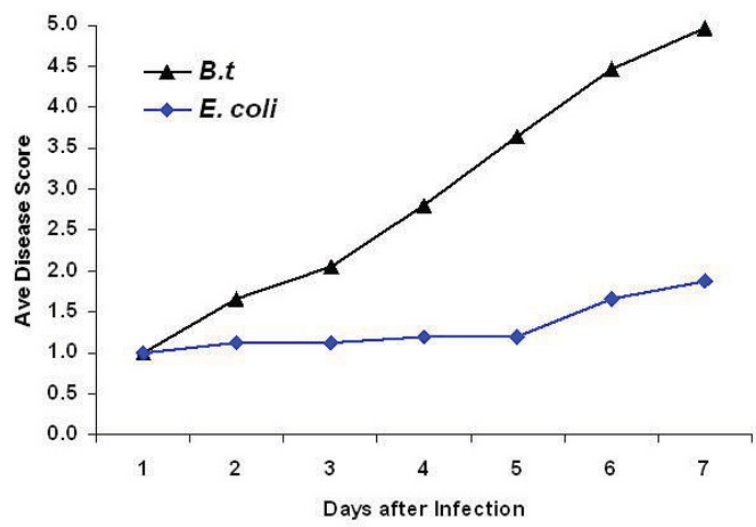

D
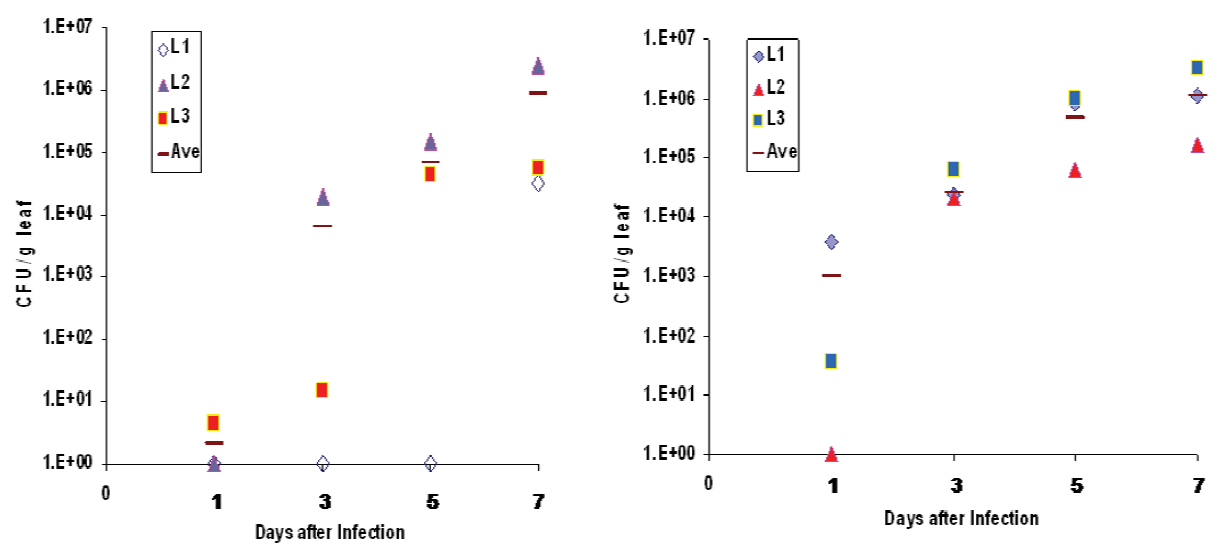

Figure $1 \mathrm{~B}$. thailandensis infection and replication in tomato plantlets. Tomato plantlets were infected with B. thailandensis and monitored over a period of seven days. On day 7, representative photographs of the uninfected plantlets (A) and the infected plantlets (B) were taken. (C) Tomato plantlets infected with $B$. thailandensis were scored daily based on the extent of disease symptoms on an index from $1-5$ over a period of seven days. The average score was calculated based on at least 100 plantlets cumulative from several experiments. (D) Each graph represents bacterial counts from leaves of one $B$. thailandensis infected plantlet over days 1, 3, 5 and 7 . Each symbol $L$ represents one leaf. 


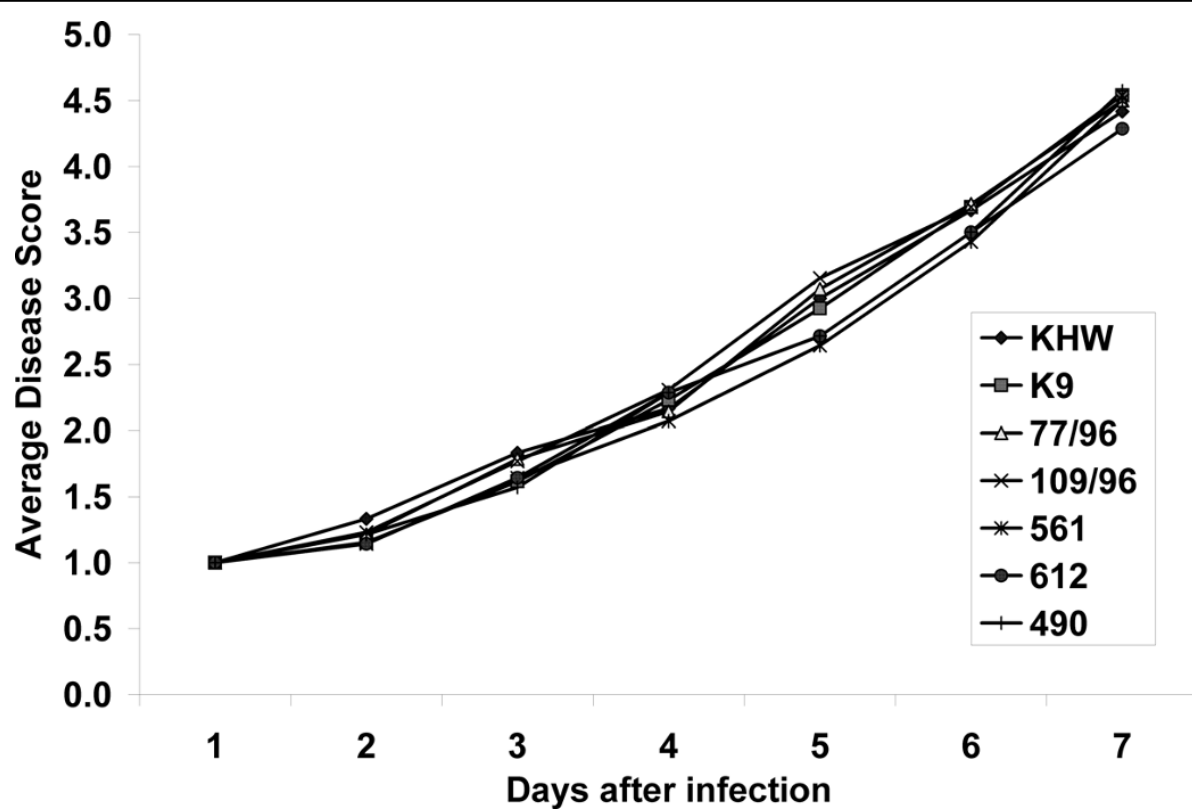

Figure 2 Infection of tomato plantlets with different B. pseudomallei isolates. KHW and K9 (K96243) are clinical isolates, 77/96 and 109/96 are soil isolates, 561 is isolated from a kangaroo, 612 from a crown pigeon and 490 from a Bird of Paradise. The average disease score was calculated based on 12 plantlets per bacterial isolate cumulative from two experiments.

shows that T3SS1 and T3SS2 are not involved in mediating cytoxicity to mammalian cells. To exclude the possibility that any defect we see with the T3SS mutants would be due to a reduced fitness, we ascertained that all mutants grew as well as wildtype bacteria in LB and plant MS medium (Fig 4B-C). However, infection of tomato plantlets via unwounded roots showed that plants infected by the T3SS1 and T3SS2 mutants exhibited significant delay in disease compared to plants infected by wildtype bacteria (Fig 4D). Statistical analysis of the average disease score over 7 days showed that the T3SS1, 2 and 3 mutants were significantly less virulent from the wildtype bacteria $(p<0.001)$. T3SS1 and T3SS2 mutants were also significantly less virulent compared to the T3SS3 mutant $(p<0.001)$. This shows that both T3SS1 and T3SS2 contribute significantly to pathogen virulence towards tomato plants. The T3SS3 mutant also showed an intermediate degree of virulence between wildtype bacteria and the T3SS1 and T3SS2 mutants, likely because T3SS3 has a non-redundant role in mediating virulence in the susceptible tomato plants.

Susceptibility of rice and Arabidopsis plantlets to $B$. pseudomallei and $B$. thailandensis infection

Both B. thailandensis and B. pseudomallei did not cause any discernible symptoms in rice plantlets when infected via roots (unwounded or wounded) nor via inoculation through the leaves. B. thailandensis and B. pseudomallei infection of rice plantlets showed identical disease scores over 7 days (Fig 5A). We were unable to recover any bacteria from the leaves after infection via the roots. When bacteria were inoculated directly into the leaf blade, no bacteria were recoverable from the leaf one day after inoculation, indicating a lack of establishment of infection. The inoculated leaves did not show any yellowing (data not shown) as seen in the tomato leaves. Thus, rice plants are non-hosts to the bacteria. As Arabidopsis thaliana has been used extensively as a plant host model for several pathogens, we tested B. thailandensis and B. pseudomallei infection in Arabidopsis plantlets via the roots. The average disease scores were maintained at 1 and increased only slightly at days 6 and 7 and were identical for both $B$. thailandensis and B. pseudomallei infection (Fig 5B).

\section{Discussion}

B. cepacia, the important opportunistic pathogen often associated with cystic fibrosis and chronic granulomatous disease patients [21], was originally described as a phytopathogen causing soft rot in onions [22]. Subsequently, many strains from various $B$. cepacia complex were shown to be able to cause disease in the alfalfa infection model as well as in the rat agar bead model [23]. In this study, we show that B. pseudomallei and $B$. thailandensis are also potential plant pathogens. They are capable of infecting susceptible plants such as tomato.

Plant pathogenic bacteria have been shown to express a large number of T3SS effectors capable of interfering 

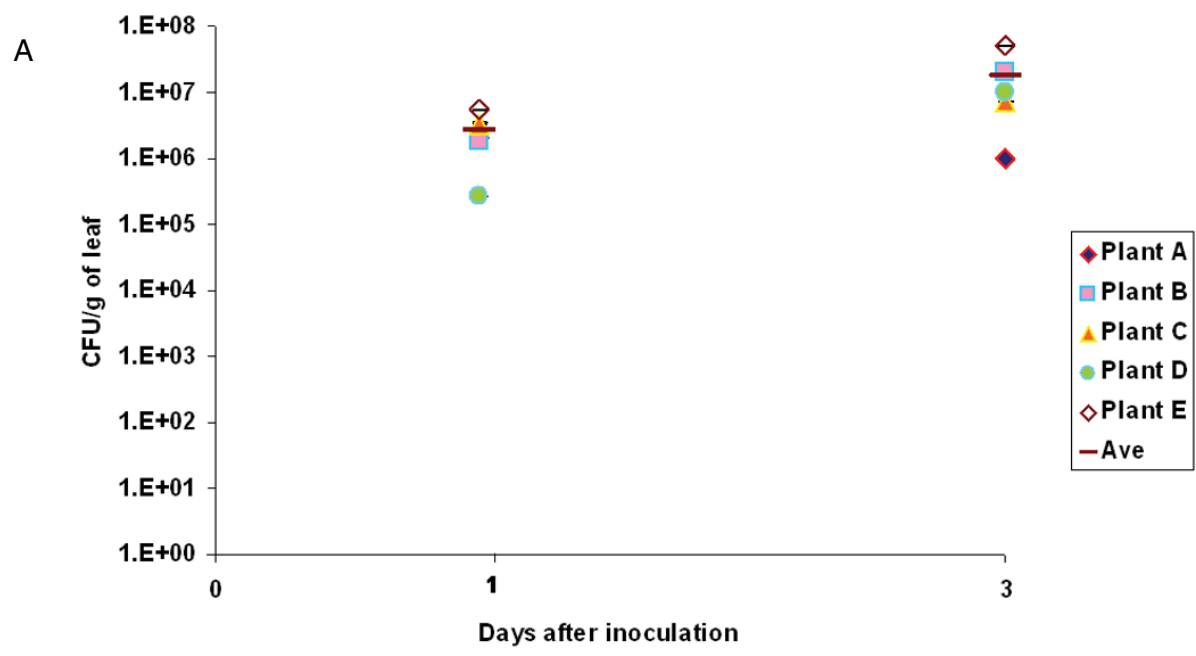

B

C

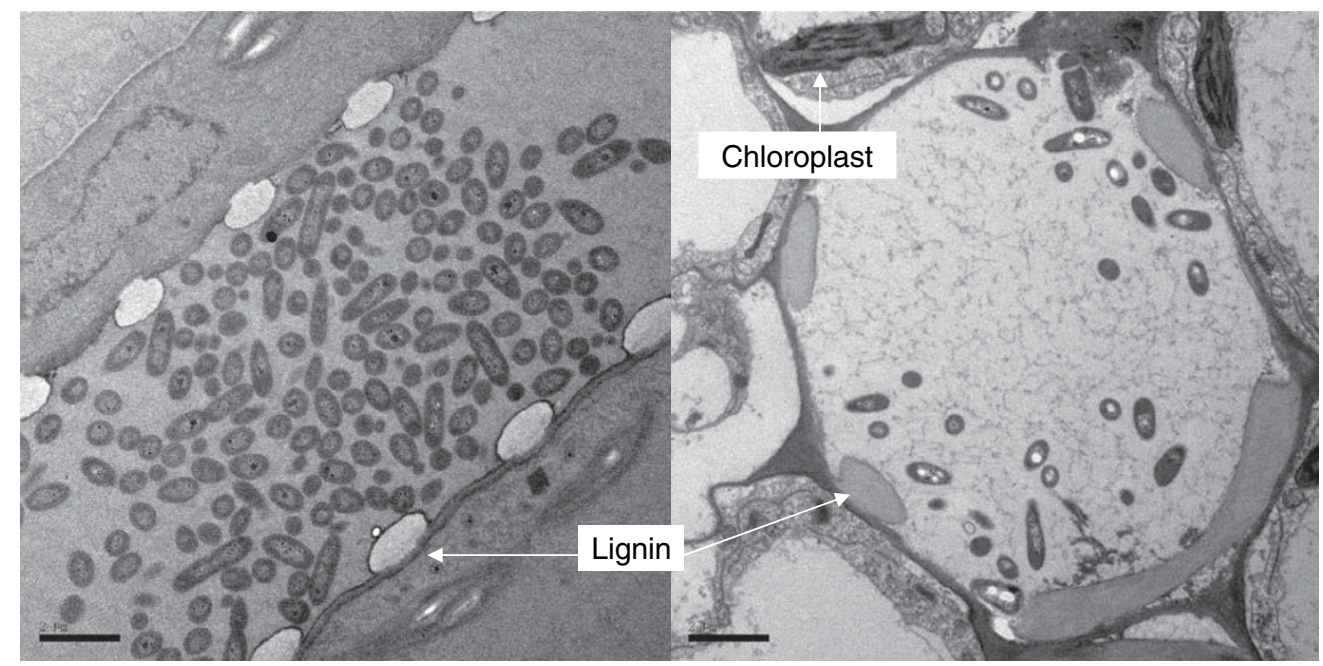

Figure 3 Replication and localization of bacteria in tomato leaves. A) B. thailandensis multiplication in tomato leaves was measured at one and three days post inoculation. The graph is representative of two separate experiments. Representative transmission electron micrographs show localization of bacteria in tomato leaf determined one day after infection. B) Leaves infected with B. thailandensis showing the longitudinal section of xylem vessel and C) leaves infected with B. pseudomallei showing the cross-sectional view. Bar represents $2 \mu \mathrm{m}$.

with plant basal defense triggered by bacterial pathogenassociated molecular patterns (PAMPs) as well as Resistance $(\mathrm{R})$ protein-mediated immunity typically characterized by the Hypersensitive Response (HR) [24-26]. The outcome of the interaction with susceptible hosts for these successful pathogens would be disease. We found that the virulence of $B$. pseudomallei in tomato is contributed significantly by T3SS1 and T3SS2, but to a much lesser extent by T3SS3. T3SS1 and T3SS2 are likely non-redundant to each other in causing disease because each mutant demonstrates significant attenuation, possibly because both T3SS1 and T3SS2 are coordinately involved in pathogenesis. This is the first time that a role has been defined for T3SS1 and T3SS2 in $B$. pseudomallei, showing that they are functional and not simply vestiges of evolution. The role of T3SS3 could be due to its contribution of a structural component or chaperone to the other two T3SS or an effector which could also interfere with plant cell physiology albeit less efficiently than with mammalian cells. Nevertheless, our study shows the important role played by T3SS in B. pseudomallei pathogenesis in tomato plants.

In contrast to tomato, we found that both B. pseudomallei and B. thailandensis are non-adapted for rice. This is not surprising as $B$. pseudomallei are routinely recovered from rice paddy fields in regions of 
A

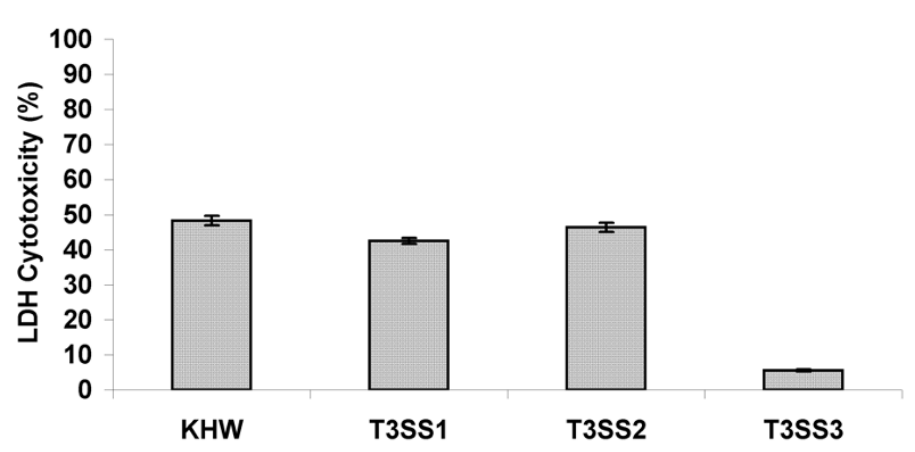

B

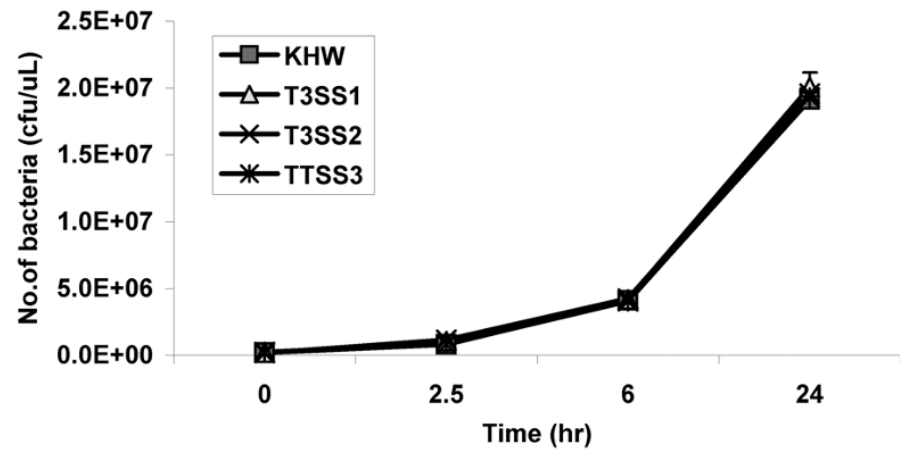

C

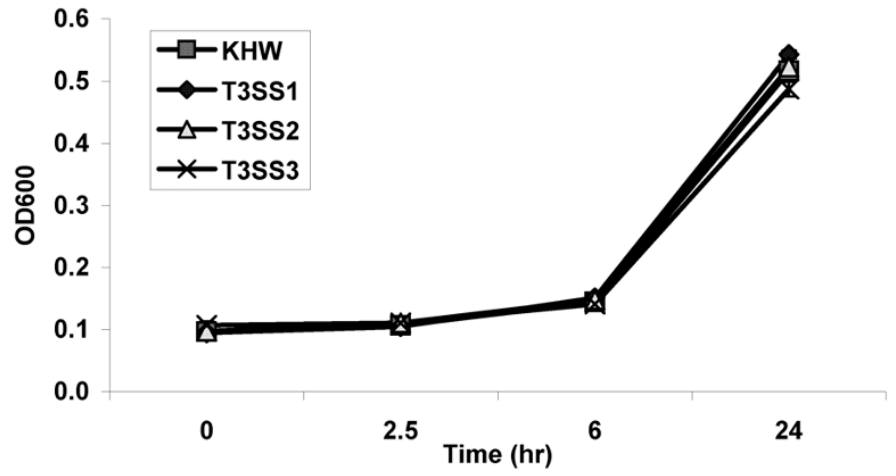

D

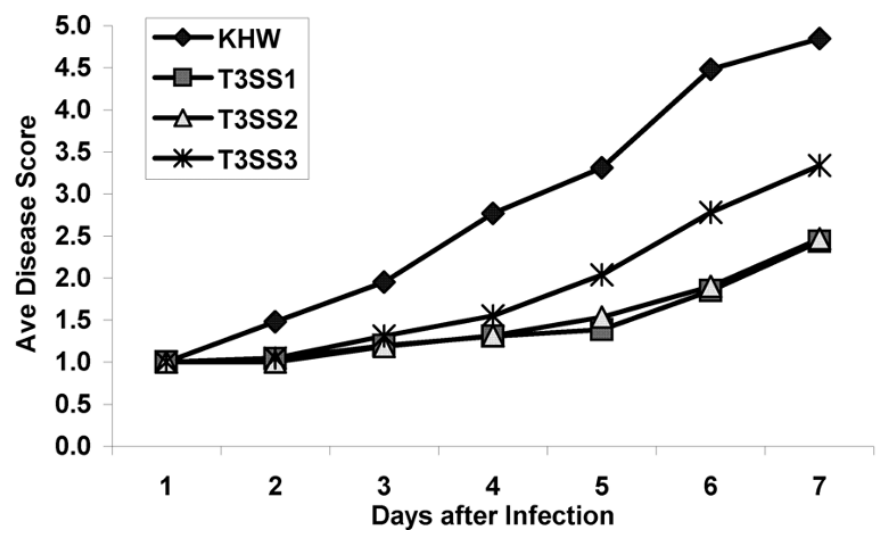

Figure 4 The role of T3SS in plant infection. (A) Cytotoxicity of wild-type B. pseudomallei and its T3SS mutants on THP-1 cells infected for six hours at an MOI of 100:1. Growth of B. pseudomallei and its T3SS mutants in LB (B) and MS (C) media. The graph is representative of two separate experiments. (D) Virulence of wildtype B. pseudomallei and its T3SS mutants on tomato plantlets. The average disease score with standard deviation is calculated based on at least 100 plantlets cumulative from several experiments. 
A

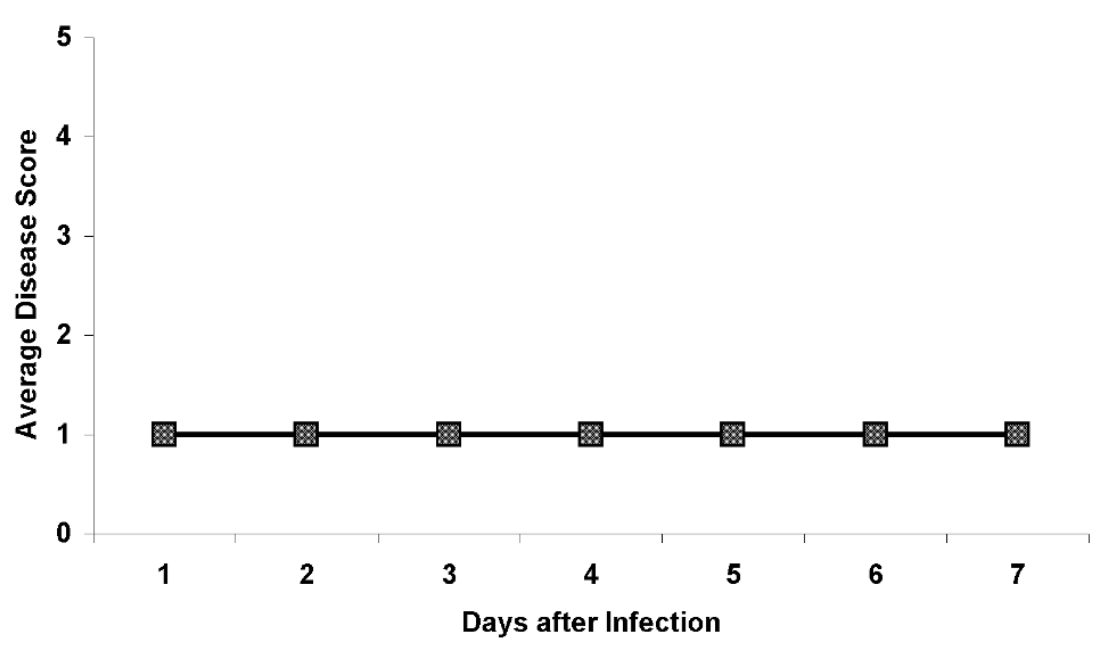

B

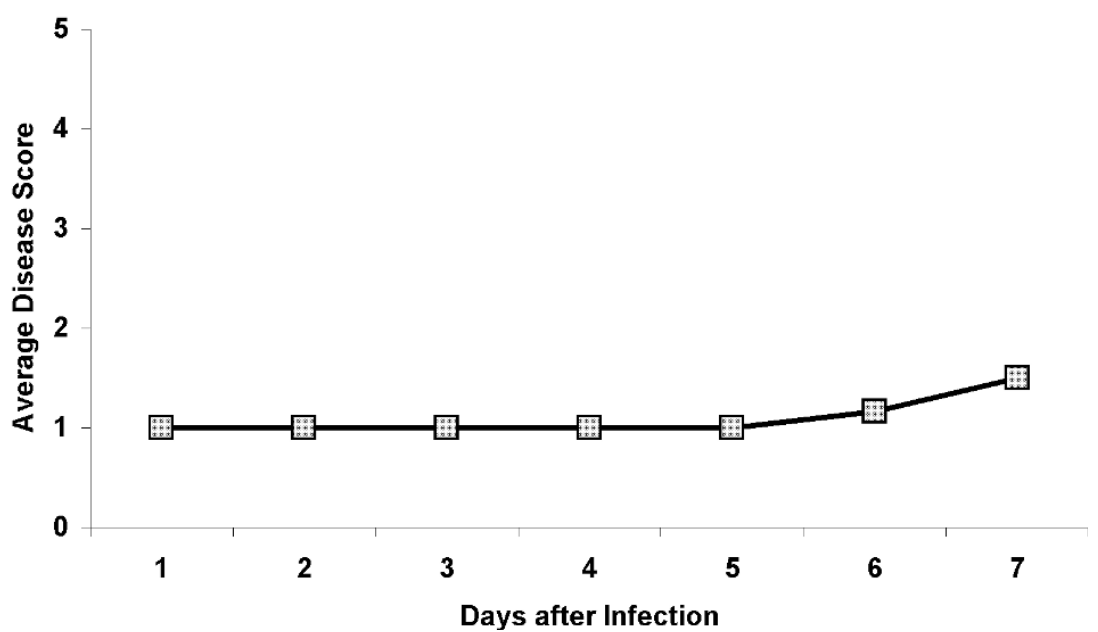

Figure 5 B. pseudomallei and B. thailandensis infection of rice (A) and Arabidopsis (B) plantlets. Each graph represents an experiment of 6 plantlets infected either with $B$. pseudomallei or $B$. thailandensis as both types of infections resulted in identical disease scores. Each experiment with $B$. pseudomallei or $B$. thailandensis infection had been repeated twice.

endemicity such as Thailand and have never been reported to cause any disease in rice plants. It is possible that PAMPs from B. pseudomallei and B. thailandensis are able to trigger an effective basal defence from rice to halt bacterial colonization, a common means of plant resistance against non-adapted microorganisms [24-26]. Another intriguing possibility is that compounds secreted by rice plants may inhibit the growth of $B$. thailandensis and B. pseudomallei. The presence of secondary metabolites induced by B. pseudomallei infection in plants with differential susceptibility to disease could reveal novel anti-infective compounds against melioidosis to counter the problem of extensive antibiotic resistance in this bacterium.
Thus, B. pseudomallei joins a growing list of human pathogens which have been found to be able to infect plants [27], the first of which to be described was $P$. aeruginosa [28]. The plant host model has been used to perform large scale screening of a library of $P$. aeruginosa mutants to identify novel virulence factors [29] as some virulence factors encoded by genes such as tox $A$, $p l c S$ and $g a c A$ were shown to be important for bacterial pathogenesis in both plants and animals [6]. Given the evidence that B. pseudomallei T3SS3 may be capable of interacting with both mammalian and plant hosts, and the ability of $B$. pseudomallei to infect tomato, one could develop susceptible plants as alternative host models for large scale screening of B. pseudomallei 
mutants to aid in novel virulence factor discovery, similar to what had been done for $P$. aeruginosa.

Previously, B. pseudomallei has been shown to infect C. elegans [30] and Acanthamoeba species [31] and C. elegans could be used as an alternative host model for large scale screening and identification of B. pseudomal$l e i$ virulence factors [30]. Our current finding reveals the additional versatility of $B$. pseudomallei as a pathogen and further research would likely uncover novel bacterial mechanisms capable of interacting with its varied hosts. Much more work is needed to define the susceptibility of various plant species to B. pseudomallei to find a suitable plant host for virulence factor discovery. It remains to be seen if $B$. pseudomallei is a natural pathogen for crops such as tomatoes.

\section{Conclusions}

In summary, we identified B. pseudomallei as a plant pathogen capable of causing disease in tomato but not rice plants. B. pseudomallei T3SS1 and T3SS2 contribute significantly to disease whereas T3SS3 plays a more minor role. Although the significance of $B$. pseudomallei as a natural plant pathogen in the environment is unknown, one could postulate that certain plants may serve as a reservoir for the bacteria. Since B. pseudomal$l e i$ is classified as a bioterrorism agent by the US Centers for Disease Control and Prevention http://www.cdc. gov/od/sap, our findings indicate that it may be necessary to re-evaluate whether $B$. pseudomallei poses threats beyond the animal kingdom and whether plant systems could be used as environmental indicators of the presence of the bacteria either as endemic residents or due to the intentional release by terrorists, a concept that has been previously proposed [27].

\section{Acknowledgements}

This work was funded by the grant 04/1/21/19/329 from the Singapore Biomedical Research Council (BMRC). We thank Chiang Shiong Loh for providing Arabidopsis seeds. We also thank Seng Kee Tan for technical advice on plant infection. YHL was funded by a stipend from Temasek Polytechnic.

\section{Author details \\ 'Department of Biochemistry, Yong Loo Lin School of Medicine, National University of Singapore, 8 Medical Drive, 117597, Singapore. ${ }^{2}$ Immunology Program, National University of Singapore, 28 Medical Drive, 117456, Singapore. ${ }^{3}$ Microscopy and Imaging Facilities, Temasek Life Sciences Laboratory, 1 Research Link, 117604, Singapore.}

\section{Authors' contributions}

YHL participated in the design of the study, carried out most experiments, analyzed and interpreted the data, and performed statistical analysis. YC generated molecular tools and some bacterial mutants. XO performed the TEM. YHG conceived of the study, participated in its design and interpretation of data and wrote the manuscript. All authors read and approved the final manuscript.

Received: 10 September 2009

Accepted: 29 January 2010 Published: 29 January 2010
References

1. Currie BJ, Fisher DA, Howard DM, Burrow JNC, Lo D, Selva-nayagam S, Anstey NM, Huffam SE, Snelling PL, Marks PJ, Stephens DP, Lum GD, Jacups SP, Krause VL: Endemic melioidosis in tropical northern Australia: A 10-year prospective study and review of literature. Clin Infect Dis 2000, 31:981-986.

2. Leelarasamee A: Melioidosis in southeast asia. Acta Trop 2000, 74:129-132

3. Sprague LD, Neubauer $\mathrm{H}$ : Melioidosis in animals: $\mathrm{A}$ review on epizootiology, diagnosis and clinical presentation. J Vet Med 2004, 51:305-320.

4. Leelarasamee A, Bovornkitti S: Melioidosis: review and update. Rev Infect Dis 1989, 11:413-425

5. Leelarasamee A: Recent development in melioidosis. Curr Opin Infect Dis 2004, 17:131-136.

6. Dance DA: Melioidosis: the tip of the iceberg?. Clin Microbiol Rev 1991, 4:52-60.

7. Holden MTG, Titball RW, Peacock SJ, Cerdeno-Tarraga AM, Atkins T, Crossman LC, Pitt T, Churcher C, Mungall K, Bentley SD, Sebaihia M, Thomson NR, Bason N, Beacham IR, Brooks K, Brown KA, Brown NF, Challis GL, Cherevach I, Chillingworth T, Cronin A, Crosset B, Davis P, DeShazer D, Feltwell T, Fraser A, Hance Z, Hauser H, Holroyd S, Jagels K, Keith KE, Maddison M, Moule S, Price C, Quail MA, Rabbinowitsh E, Rutherford K, Sanders M, Simmonds M, Songsivilai S, Stevens K, Tumapa S, Vesaratchavest M, Whitehead S, Yeats C, Barrell BG, Oyston PCF, Parkhill J: Genomic plasticity of the causative agent of melioidosis, Burkholderia pseudomallei. Proc Natl Acad Sci USA 2004, 101:14240-14245.

8. Attree O, Attree I: A second type III secretion system in Burkholderia pseudomallei: who is the real culprit?. Microbiology 2001, 147:3197-3199.

9. Rainbow L, Hart CA, Winstanley C: Distribution of type III secretion gene clusters in Burkholderia pseudomallei, B. thailandensis and B. mallei. J Med Microbiol 2002, 51:374-384.

10. Stevens MP, Haque A, Atkins T, Hill J, Wood MW, Easton A, Nelson M, Underwood-Fowler C, Titball RW, Bancroft GJ, Galyov EE: Attenuated virulence and protective efficacy of a Burkholderia pseudomallei bsa type III secretion mutant in murine models of melioidosis. Microbiology 2004, 150:2669-2676.

11. Winstanley C, Hales BA, Hart CA: Evidence for the presence in Burkholderia pseudomallei of a type III secretion system-associated gene cluster. J Med Microbiol 1999, 48:649-656.

12. Warawa J, Woods DE: Type III secretion system cluster is required for maximal virulence of Burkholderia pseudomallei in a hamster infection model. FEMS Microbiol Lett 2005, 242:101-108.

13. Brett PJ, Deshazer D, Woods DE: Characteristics of Burkholderia pseudomallei and Burkholderia pseudomallei-like strains. Epidemiol Infect 1997, 118:137-148.

14. Smith MD, Angus BJ, Wuthiekanun V, White NJ: Arabinose assimilation defines a nonvirulent biotype of Burkholderia pseudomallei. Infect Immun 1997, 65:4319-4321.

15. Tans-Kersten J, Huang H, Allen C: Ralstonia solanacearum needs motility for invasive virulence on tomato. J Bacteriol 2001, 183:3597-3605.

16. Spurr AR: A low-viscosity epoxy resin embedding medium for electron microscopy. J Ultrastruct Res 1969, 26:31-43.

17. Murashige T, Skoog F: A revised medium for rapid growth and bioassays with tobacco tissue culture. Physiol Plant 1962, 15:473-497.

18. Chan YH: Biostatics 301. Repeated measurement analysis. Singapore Med J 2004, 45:354-369.

19. Agrios GN: Plant pathology. Elsevier Academic Press, Fifth 2005

20. Sun GW, Lu JH, Pervaiz S, Cao WP, Gan YH: Caspase-1 dependent macrophage death induced by Burkholderia pseudomallei. Cell Microbiol 2005, 7:1447-1458.

21. Coenye T, Vandamme P: Diversity and significance of Burkholderia species occupying diverse ecological niches. Environ Microbiol 2003, 5:719-729.

22. Burkholder $\mathrm{WH}$ : Sour skin, a bacteria rot of onion bulbs. Phytopathology 1950, 40:115-117.

23. Bernier SP, Silo-Suh L, Woods DE, Ohman DE, Sokol PA: Comparative analysis of plant and animal models for characterization of Burkholderia cepacia virulence. Infect Immun 2003, 71:5306-5313.

24. Abramovitch RB, Anderson JC, Martin GB: Bacterial elicitation and evasion of plant innate immunity. Nat Rev Mol Cell Biol 2006, 7:601-611.

25. Gohre V, Robatzek S: Breaking the Barriers: Microbial Effector Molecules Subvert Plant Immunity. Annu Rev Phytopathol 2008, 46:189-215. 
26. Cui H, Xiang T, Zhou JM: Plant immunity: a lesson from pathogenic bacterial effector proteins. Cell Microbiol 2009, 11:1453-1461.

27. Prithiviral B, Weir T, Bais HP, Schweizer HP, Vivanco JM: Plant models for animal pathogenesis. Cell Microbiol 2005, 7:315-324.

28. Rahme LG, Stevens EJ, Wolfort SF, Shao J, Tompkins RG, Ausubel FM: Common virulence factors for bacterial pathogenicity in plants and animals. Science 1995, 268:1899-1901.

29. Rahme LG, Tan M-W, Le L, Wong SM, Tompkins RG, Calderwood SB, Ausubel FM: Use of model plant hosts to identify Pseudomonas aeruginosa virulence factors. Proc Natl Acad Sci USA 1997, 94:13245-13250.

30. Gan YH, Chua KL, Chua HH, Liu B, Hii CS, Chong HL, Tan P:

Characterization of Burkholderia pseudomallei infection and identification of novel virulence factors using a Caenorhsbditis elegans host system. Mol Microbiol 2002, 44:1185-1197.

31. Inglis TJJ, Rigby P, Robertson TA, Dutton NS, Henderson M, Chang BJ: Interaction between Burkholderia pseudomallei and Acanthamoeba species results in coiling phagocytosis, endamebic bacterial survival and escape. Infect Immun 2000, 68:1681-1686.

32. Schäfer A, Tauch A, Jäger W, Kalinowski J, Thierbach G, Pühler A: Small mobilizable multi-purpose cloning vectors derived from the Escherichia coli plasmids pK18 and pK19: selection of defined deletions in the chromosome of Corynebacterium glutamicum. Gene 1994, 145:69-73.

33. Simon $R$, Priefer $U$, Pühler $A$ : A broad range mobilization system for in vitro genetic engineering: Transposon mutagenesis in Gram-negative bacteria. Bio/Technology 1983, 1:784-791.

doi:10.1186/1471-2180-10-28

Cite this article as: Lee et al:: Identification of tomato plant as a novel host model for Burkholderia pseudomallei. BMC Microbiology 2010 10:28. 